\title{
Evaluating statistical learning methods for cell type classification and feature selection using RNA-seq data
}

Hao Chen

From UT-KBRIN Bioinformatics Summit 2014

Cadiz, KY, USA. 11-13 April 2014

\section{Background}

Single cell RNA-seq offers the opportunity to develop a systematic inventory of cell types in the brain.

\section{Materials and methods}

We evaluated several statistical learning methods for classifying RNA-seq data using samples obtained from different brain regions as surrogates for single cell data. These include the ventral tegmental area (VTA, $n=36$ ), the nucleus accumbens core $(\mathrm{AcbC}, \mathrm{n}=34)$ and shell (AcbS, $\mathrm{n}=30$ ) [1]. We tested hierarchical clustering methods using different distance matrices (correlation, uncentered, abscor) and clustering algorithms. Multiscale bootstrap was used to evaluate the statistical significance of the clusters. We also tested the Bayesian Hierarchical Clustering method, which uses hypothesis testing to decide which cluster mergers increase the tree quality and calculates the optimum tree depth.

\section{Results}

Both the Ward's algorithm and the Bayesian method correctly classified the majority of the samples. The reliability of the clusters were evaluated by repeated down sampling (without replacement). We found that sample sizes of 4-8/cluster were classified by the Ward's method with a very low error rate $(0.02)$, while the error rate of BHC was slightly higher (0.11). We then compared Lasso and elastic net regularized logistic regression on the selection of genes that can be used as the molecular identity of each cluster. The Lasso method identified 15 genes (including Th, tyrosine hydroxylase) for the VTA cluster with cross validation error of 0.014 .

Correspondence: hchen@uthsc.edu

Department of Pharmacology, University of Tennessee Health Science Center, Memphis, TN 38106, USA
1. Chen H, Luo R, Gong S, Matta SG, Sharp BM: Protection genes in nucleus accumbens shell affect vulnerability to nicotine self-administration across isogenic strains of adolescent rat. PLoS One 2014, 9(1):e86214

doi:10.1186/1471-2105-15-S10-P26

Cite this article as: Chen: Evaluating statistical learning methods for cell type classification and feature selection using RNA-seq data. BMC Bioinformatics 2014 15(Suppl 10):P26. 